\title{
Tetracycline Sclerotherapy in the Management of Chyle Leak
}

\author{
${ }^{1}$ Madhumati Singh, ${ }^{2} \mathrm{~S}$ Vijayanand, ${ }^{3} \mathrm{~B}$ Amit, ${ }^{4}$ Ragesh Raman, ${ }^{5} \mathrm{R}$ Narahari
}

\begin{abstract}
Chyle leak following neck dissection is a rare but recognized complication with significant morbidity. The timing, indications and treatment modalities of chylous fistula remain controversial. Sclerotherapy using tetracycline is not a much acclaimed form of treatment in the management of chylous fistula. We report a case of postoperative chylous leak successfully managed by injection of tetracycline into the supraclavicular wound bed.
\end{abstract}

Keywords: Chyle leak, Chylous fistula, Neck dissection, Sclerotherapy, Tetracycline.

How to cite this article: Singh M, Vijayanand S, Amit B, Raman R, Narahari R. Tetracycline Sclerotherapy in the Management of Chyle Leak. J Health Sci Res 2015;6(1):14-16.

Source of support: Nil

Conflict of interest: None

\section{INTRODUCTION}

Chylous fistula, secondary to intraoperative damage to the thoracic duct or the right lymphatic duct, is a known but rare complication of head-and-neck surgery affecting $1-3 \%$ of neck dissections, especially the left side. It occurs due to the thin walled, fragile nature of the duct and the anatomical variations in its termination into the junction between the left internal jugular vein and subclavian vein. ${ }^{1}$ Cheever in 1875 recorded the first case of operative damage to the thoracic duct. ${ }^{2}$

Chyle leak is a potentially life-threatening condition and a high output fistula can lead to protein loss, fluid and electrolyte disturbance, immunosuppression, chylothorax, wound breakdown and sepsis., ${ }^{3,4}$ Accumulation of chyle within the neck can result in dermal irritation, erythema, dehiscence of skin flaps and even great vessel compromise. ${ }^{2}$ We present a case of chyle leak as a complication of modified radical neck dissection in a patient with oral squamous cell carcinoma which was successfully managed by tetracycline sclerotherapy.

\footnotetext{
${ }^{1}$ Professor and Head, ${ }^{2-5}$ Postgraduate

${ }^{1-5}$ Department of Oral and Maxillofacial Surgery, Rajarajeswari Dental College and Hospital, Bengaluru, Karnataka, India

Corresponding Author: S Vijayanand, Postgraduate Department of Oral and Maxillofacial Surgery, Rajarajeswari Dental College and Hospital, Bengaluru, Karnataka, India Phone: +918904304748, e-mail: drsvijayanand@gmail.com
}

\section{CASE REPORT}

A 40 years old female patient presented in our Department with a 6 months history of a slowly enlarging lesion in the left maxillary alveolus region. Physical examination showed a $1-1.5 \mathrm{~cm}$ ulcerating mass arising between the left maxillary lateral incisor and the first premolar region. Neck examination revealed a palpable left level II cervical lymph node. Incisional biopsy showed a moderately differentiated invasive squamous cell carcinoma (SCC). Staging computed tomography (CT) imaging of the head, neck and chest also revealed involvement of level II lymph node. Primary tumor excision with left-sided modified radical neck dissection to clear lymph node levels I-V was performed. Primary tumor site was reconstructed with nasolabial flap (Fig. 1).

The surgical procedure was uneventful, apart from a left-sided high output chyle leak of more than 1000 $\mathrm{ml} / 24$ hours noted postoperatively which continued even on the 2 nd day. Conservative management in the form of pressure dressing was planned following which, she continued to have a low flow chyle leak. This resulted in a cystic swelling in the base of the neck. It was treated by aspiration of the swelling followed by sclerotherapy using tetracycline (Fig. 2). A $5 \mathrm{ml}$ of $5 \%$ tetracycline solution was injected into the cystic swelling immediately after aspiration of the cyst contents. The procedure was repeated three times on alternate days and the patient continues to remain symptom free without major complications except for local pain at the site for several days.

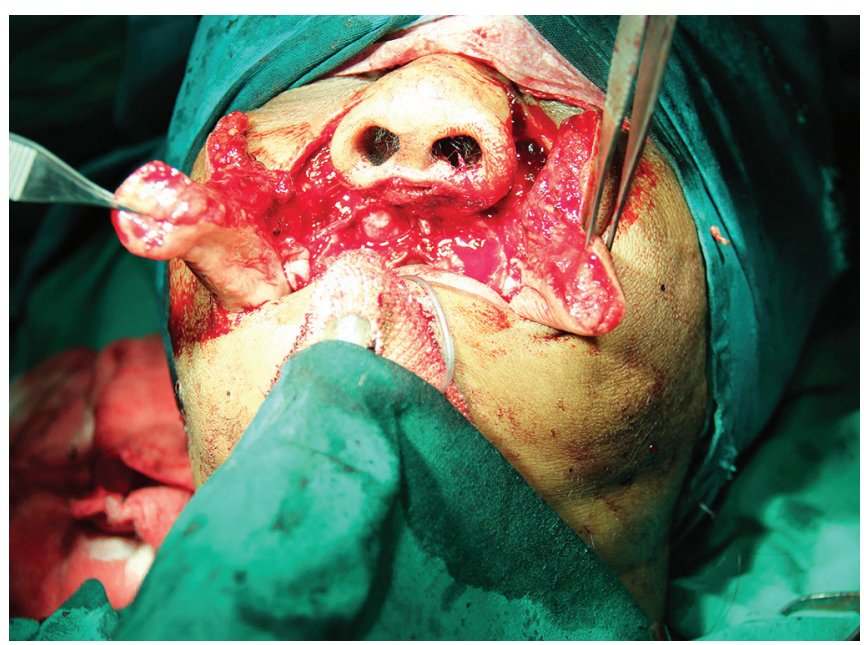

Fig. 1: Primary tumor site reconstruction done with nasolabial flap 


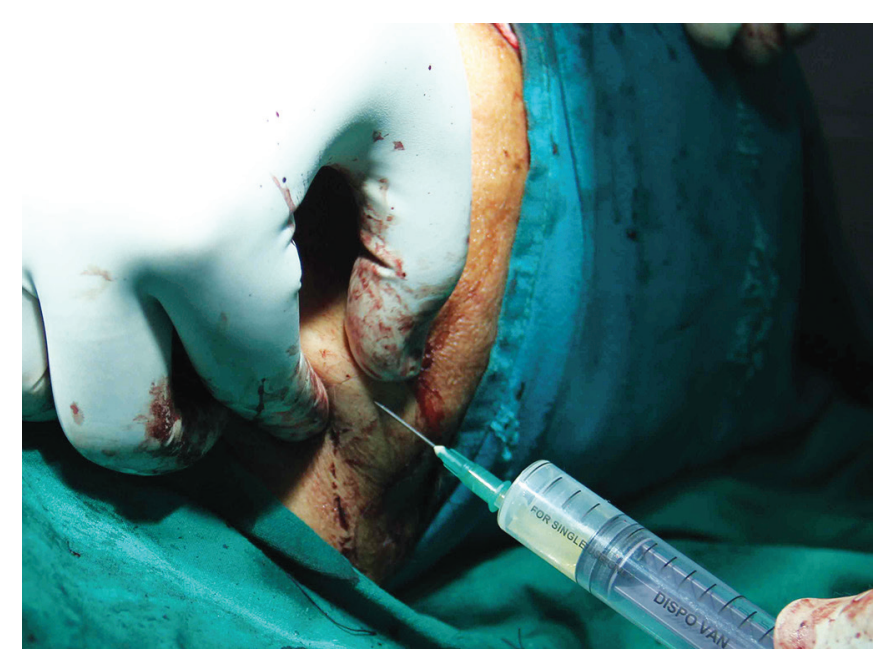

Fig. 2: Aspiration of the swelling followed by sclerotherapy using tetracycline injection

\section{DISCUSSION}

Intraoperative damage to the thoracic duct with chyle extravasation can pose a great challenge to the surgeon due to the potential risk of serious morbidity. ${ }^{2}$ High output leaks (more than 1-2 liters/day) for more than 5-7 days duration needs active management. ${ }^{2}$ The ideal time to identify and manage thoracic duct damage is at the time of neck dissection though it may be difficult as a result of decreased volume of chyle in the thoracic duct due to preoperative fasting. ${ }^{2}$ During the dissection procedure, one should periodically inspect this area for a possible chylous leak (Fig. 3). It is good practice in radical neck dissections, to check for chyle fistulas by putting the patient in the Trendelenburg's position and by increasing intrathoracic pressure via a Valsalva manoeuvre. ${ }^{1,2}$ Once identified, repair or ligation of the damaged thoracic duct is performed. Ligation with a nonabsorbable suture is usually done using metal clips and over sewing and a local muscle like part of the sternomastoid, scalenus medius, omohyoid muscle flap or pectoralis major flap can be used., ${ }^{1,2}$ Local application of fibrin glue or cyanoacrylate tissue glue has been successfully used in the management of chyle leaks identified intraoperatively. ${ }^{2}$

Postoperative chyle leak manifests as a swelling in the neck or an unexpectedly high drain output especially on resuming feeding. ${ }^{5}$ The presence of creamy white fluid in the drain should also raise suspicion. Analysis of the drain fluid for the presence of triglycerides (>100 mg/dl) and triglyceride ratios confirms the diagnosis. ${ }^{6}$

Imaging modalities like high resolution ultrasound and CT with contrast may be used to identify the leak. Standard lymphangiography can demonstrate the foci of leak more frequently but is technically difficult. Lymphoscintigraphy using technetium 99 m-labeled filtered

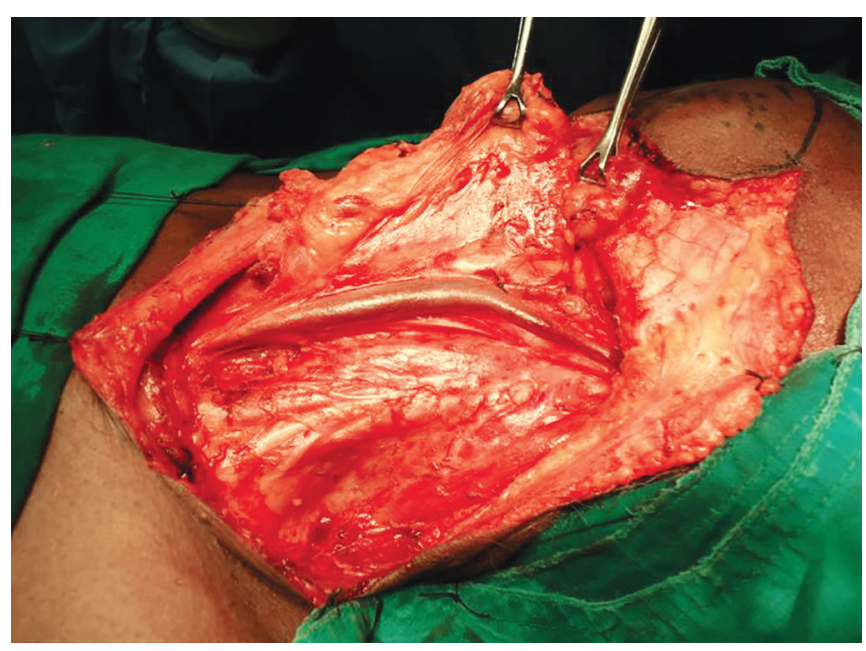

Fig. 3: Intraoperative image showing neck dissection

sulphur confirms chyle leak by identifying regional 'hot spots' and may be useful in surgical planning. ${ }^{2}$

Conservative management is typically used in cases with low output chyle leak (less than 1 liter/day). ${ }^{7}$ The patient is placed in an upright position with a drain coming out of the skin far laterally. ${ }^{1}$ Local pressure dressings applied to the neck and reduction of chyle production using medium-chain triglyceride (MCT) or a fat-free diet has been found to be beneficial. ${ }^{7,8}$ Pharmacological agents like somatostatin and octreotide reduce gastric, pancreatic and intestinal secretions and intestinal fat absorption thereby reducing chyle production. ${ }^{3}$ Newer agents like orlistat, a pancreatic lipase inhibitor and etilefrine, a sympathomimetic amine have also been tried. ${ }^{2}$

The injection of sclerosant agents (tetracyclines, talc powder or povidone-iodine) percutaneously with or without USG guidance or through the drainage catheter, into the supraclavicular wound bed may result in a rapid reduction in the fistula output, obviating the need for major invasive procedure. ${ }^{1,9}$ Sclerotherapy using tetracycline has been shown to be a successful method of treating chylous fistula though it has been reported rarely in literature. ${ }^{4}$

Presence of a high-output chyle leak or failure of conservative measures necessitates some form of active intervention. Percutaneous lymphangiography-guided cannulation with embolization of the thoracic duct using either coils or cyanoacrylate tissue glue is a minimally invasive alternative to open surgery. ${ }^{2,10}$ Thoracoscopic ligation of the thoracic duct has been advocated by some authors. ${ }^{11}$ With the advent of minimally invasive procedures, re-exploration of the wound with repair is rarely indicated. ${ }^{2,7}$ Local muscle flaps, such as the inferiorly based omohyoid flap with oversewing is usually sufficient to occlude the thoracic duct. ${ }^{2}$ Additionally, the use of 
fibrin glue or cyanoacrylate tissue glue is recommended as these are able to directly seal any small branches of the thin walled ductal system. ${ }^{2}$

\section{CONCLUSION}

Chyle leak post neck dissection can lead to considerable morbidity and necessitate prompt management. Identification and treatment at the time of surgery is the best management option. Sclerotherapy with tetracycline is not a well-known form of treatment in these cases. It is, however an effective adjunct in the management of chylous fistulas and should be attempted before surgical re-exploration. It is associated with minimal complications and appears to be successful in selected cases.

\section{REFERENCES}

1. Rollon A, Salazar C, Mayorga F, Marin R, Infante P. Severe cervical chyle fistula after radical neck dissection . Int J Oral Maxillofac Surg 1996;25(5):363-365.

2. Brennan PA, Blythe JN, Herd MK, Habib A, Anand R. The contemporary management of chyle leak following cervical thoracic duct damage. Br J Oral Maxillofac Surg 2012;50(3): 197-201.
3. Ilczyszyn A, Ridha H, Durrani AJ. Management of chyle leak post neck dissection: a case report and literature review. J Plast Reconstr Aesthet Surg 2011;64(9):e223-e230.

4. Srinivasa D, Green J, Pandya H, Brown A. Management of chyle leak with tetracycline sclerotherapy. Br J Oral Maxillofac Surg 2007;45(7):e7.

5. Crumley RL, Smith JD. Postoperative chylous fistula prevention and management. Laryngoscope 1976;86(6):804e13

6. Rodgers GK, Johnson JT, Petruzzelli GJ, Warty VS, Wagner RL. Lipid and volume analysis of neck drainage in patients undergoing neck dissection. Am J Otolaryngol 1992;13(5):306e9.

7. Gregor RT. Management of chyle fistulization in association with neck dissection. Otolaryngol Head Neck Surg 2000; 122(3):434-439.

8. Hashim SA, Roholt HB, Babayan VK, Vanitallie TB. Treatment of chyluria and chylothorax with medium-chain triglyceride. N Engl J Med 1964;270(15):756-761.

9. Seelig MH, Klingler PJ, Oldenburg WA. Treatment of a postoperative cervical chylous lymphocele by percutaneous sclerosing with povidone-iodine. J Vasc Surg 1998;27(6): 1148-1151.

10. Cope C. Management of chylothorax via percutaneous embolization. Curr Opin Pulm Med 2004;10(4):311-314.

11. Van Goor AT, Kröger R, Klomp HM, de Jong MA, van den Brekel MW, Balm AJ. Introduction of lymphangiography and percutaneous embolization of the thoracic duct in a step wise approach to the management of chylous fistulas. Head Neck 2007;29(11):1017-1023. 